\title{
A novel MAP3K7 splice mutation causes cardiospondylocarpofacial syndrome with features of hereditary connective tissue disorder
}

\author{
Silvia Morlino ${ }^{1}$ Marco Castori ${ }^{2} \cdot$ Chiara Dordoni $^{3} \cdot$ Valeria Cinquina $^{3} \cdot$ Graziano Santoro $^{3} \cdot$ Paola Grammatico $^{1}$. \\ Marina Venturini ${ }^{4} \cdot$ Marina Colombi $^{3} \cdot$ Marco Ritelli $^{3}$
}

Received: 7 June 2017 / Revised: 28 November 2017 / Accepted: 5 December 2017 / Published online: 21 February 2018

(c) European Society of Human Genetics 2018

\begin{abstract}
Heterozygous variants in $M A P 3 K 7$, encoding the transforming growth factor- $\beta$-activated kinase 1 (TAK1), are associated with the ultrarare cardiospondylocarpofacial syndrome (CSCFS). Specific gain-of-function variants in the same gene cause the allelic frontometaphyseal dysplasia type 2. Phenotypic series of frontometaphyseal dysplasia also comprise variants in FLNA (type 1) and two patients with a heterozygous variant in TAB2 (type 3). We report on a 7-year-old girl with CSCFS due to the novel heterozygous c.737-7A $>\mathrm{G}$ variant in MAP3K7. The identified variant generates a new splice acceptor site causing an in-frame insertion of 2 amino acid residues (p.Asn245_Gly246insValVal), as demonstrated by RNA study. The patient was originally ascertained for a presumed hereditary connective tissue disorder due to soft/dystrophic skin, extreme joint hypermobility, polyvalvular heart disease, and upper gastrointestinal dismotility. Our study confirms locus homogeneity for CSCFS, expands the mutational spectrum of MAP3K7, and adds data on the existence of a community of connective tissue disorders caused by abnormalities of the TAK1-dependent signaling pathway.
\end{abstract}

\section{Introduction}

MAP3K7 (mitogen-activated protein kinase kinase kinase 7) maps on chromosome 6q15 and encodes the transforming growth factor- $\beta$-activated kinase 1 (TAK1), a highly conserved kinase protein activated by multiple extracellular stimuli, growth factors and cytokines [1]. TAK1 mediates a wide range of biological processes such as innate immunity, angiogenesis and myocardial homeostasis [2, 3]. It forms a complex with $\mathrm{TAB} 1, \mathrm{TAB} 2$, and $\mathrm{TAB} 3$ that, in turn, modulate TAK1 activation [4]. TAK1 and its adapter

Marco Castori

m.castori@operapadrepio.it

1 Laboratory of Medical Genetics, Department of Molecular Medicine, University "La Sapienza", San Camillo-Forlanini Hospital, Rome, Italy

2 Division of Medical Genetics, IRCCS Casa Sollievo della Sofferenza, San Giovanni Rotondo (Foggia), Italy

3 Division of Biology and Genetics, Department of Molecular and Translational Medicine, University of Brescia, School of Medicine, Brescia, Italy

4 Division of Dermatology, Department of Clinical and Experimental Sciences, Spedali Civili University Hospital, Brescia, Italy protein $\mathrm{TAB} 2$ reciprocally regulate the activation of the nuclear factor-kB, AP-1 and the mitogen-activated protein (MAP) kinase signaling pathways [5-7]. MAP3K7 heterozygous variants are related to cardiospondylocarpofacial syndrome (CSCFS) and frontometaphyseal dysplasia (FMD) type 2 (FMD2) $[8,9]$. The former is an ultrarare condition described in three sporadic and one familial cases so far $[8,10]$. FMD2 is a distinct disorder, overlapping FMD type 1 due to FLNA mono-allelic variants $[9,11]$. The MAP3K7 recurrent missense c. $1535 \mathrm{C}>\mathrm{T}$ (p.(Pro512Leu)) variant was also found in a single individual with intellectual disability [12]. Different molecular mechanisms underlie such apparently divergent phenotypes, as CSCFS is caused by non-recurrent missense variants or in-frame deletions, while FMD2 is generated by recurrent, gain-offunction variants [9]. The evidence that $M A P 3 K 7, F L N A$, and $T A B 2$ interact during morphogenesis lies on the FMD allelic series linked to presumably gain-of-function variants in either of these genes $[9,11,13]$.

Here we report a 7-year-old sporadic patient with CSCFS, due to a de novo splicing variant in MAP3K7. The patient was originally ascertained for multisystem connective tissue abnormalities and facial features resembling a previously described patient with a $T A B 2$ frameshift variant [14]. 

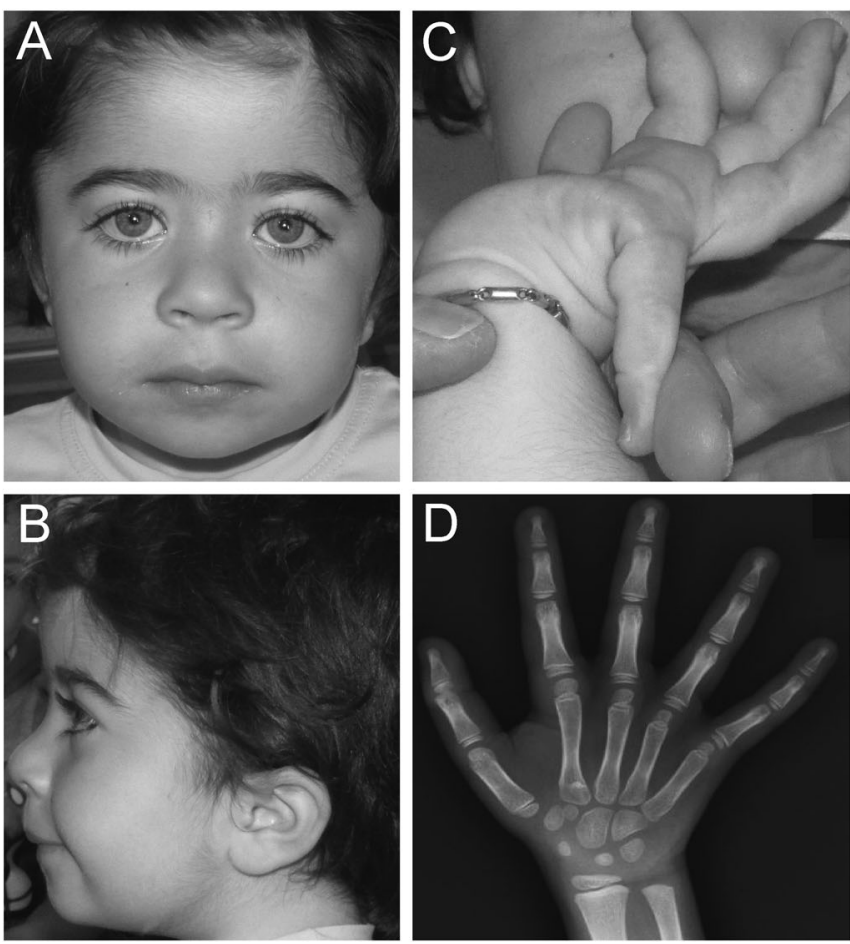

Fig. 1 a Frontal view of the patient at 4 years showing bilateral palpebral ptosis with epicanthal folds, light blue sclera, broad nasal bridge, bulbous nose with anteverted nostrils, long philtrum and wide mouth. b Lateral view of the patient showing posteriorly rotated low-set ears and hypoplastic nasal tip. c Hypermobility of the fifth finger with the fingertip touching the dorsal aspect of the forearm. d Absence of carpal fusions at X-rays of the hand. e Spine

\section{Subjects and methods}

The patient was enrolled from the routine clinical activity of the involved institutions. Institutional Review Board approval was therefore not required. The family gave written informed consent for genetic testing, publication of clinical pictures, and authorized the processing of personal data according to the Italian bioethics laws. Variant screening was performed by PCR amplification of all coding exons/ intron boundaries of TAB2 (NG_021386.1, NM_015093.5) and MAP3K7 (NG_011966.2, NM_145331.2) and subsequent sequence analysis was carried out by Sanger sequencing. For splice site prediction, the interactive biosoftware Alamut Visual version 2.9.0 was used. To verify the effect of the splice variant, reverse transcriptase-PCR (RT-PCR) was carried out by standard procedures on RNA extracted from patient's whole blood and stabilized in Paxgene tubes (PreAnalytiX). Hence, amplification of cDNA covering exons 6-8 of MAP3K7 was performed. The identified variant affecting function of $M A P 3 K 7$ was submitted to the LOVD database (URL: https://databases.lovd.nl/sha red/genes/MAP3K7; patient ID: \#00132079).
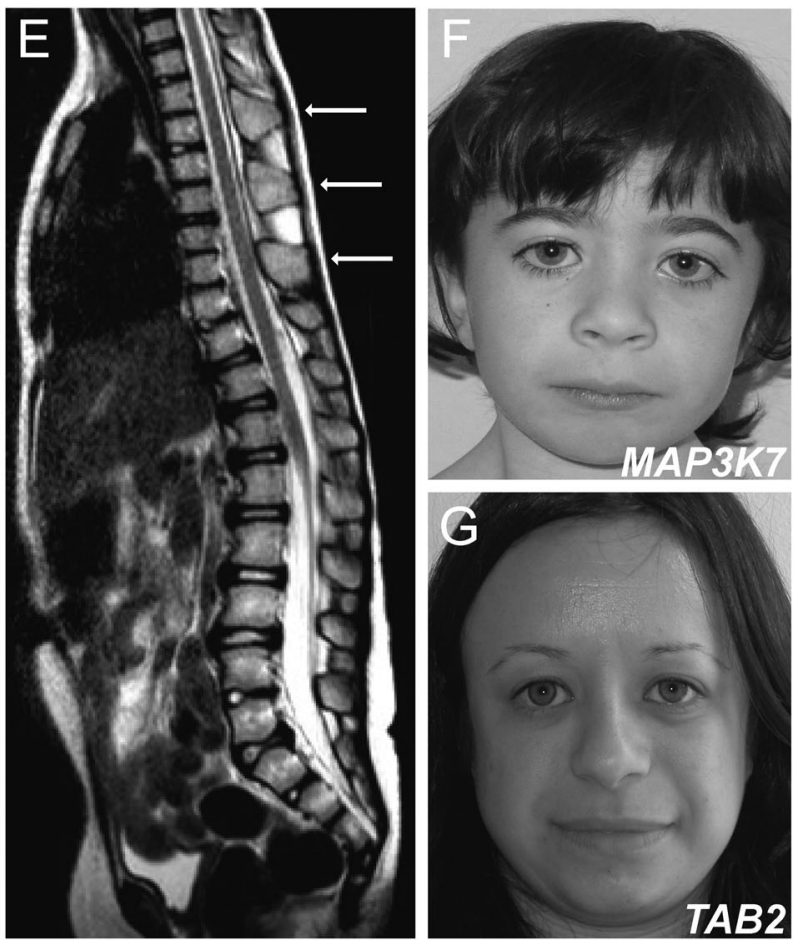

MRI at 6 years demonstrates fusion of the posterior arches of T5-T6, T7-T8, and T9-T10 (additional fusions involved the posterior arches of $\mathrm{C} 6-\mathrm{C} 7$ and $\mathrm{T} 3-\mathrm{T} 4$; not visible in this picture), and hypoplastic lower sacral vertebrae and coccyx. f Frontal view of the patient at 7 years. g For comparison, frontal view of Patient 2 from ref. [14]. with a heterozygous frameshift variant in TAB2 (color figure online)

\section{Results}

\section{Clinical report}

The patient was originally assessed for a suspected hereditary connective tissue disorder. She was born at term by Cesarean section and was the second child of healthy, nonconsanguineous parents. Third trimester prenatal ultrasound revealed reduced fetal movements. Birth weight was $2900 \mathrm{~g}$ (5-10th centile), Apgar index $7^{1} / 9^{5}$, while length and occipitofrontal circumference (OFC) were unknown. Heart ultrasound at birth and subsequent follow-ups revealed patent foramen ovale with left-right shunt and two small muscular ventricular septal defects, mitral and tricuspid valves dysplasia and mild, non-progressive aortic arch hypoplasia. Early after birth, the patient presented failure to thrive due to severe gastroesophageal reflux. Laparoscopic gastropexy at 6 months failed to improve the reflux and percutaneous endoscopic gastrostomy was performed at 4 years. At 6 years, videofluoroscopic swallowing study disclosed gastroparesis and antral fibrotic strictures. Gastrostomy was removed at 6.5 years. Psychomotor 

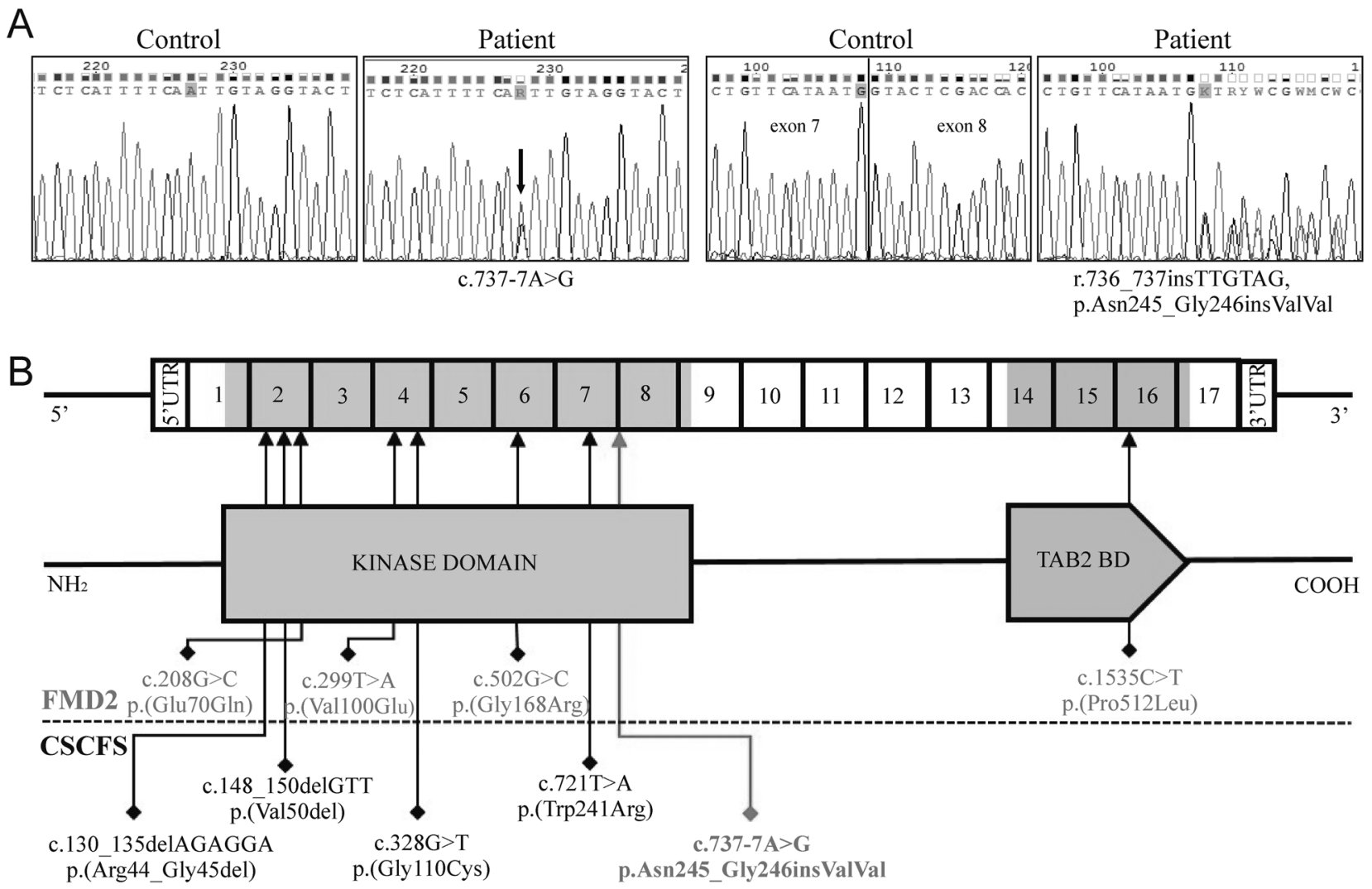

Fig. 2 a Sequence chromatograms showing the position (arrow) of the novel de novo c.737-7A $>\mathrm{G}$ variant that was detected at the heterozygous state in intron 7 of MAP3K7. Right chromatograms refer to the RT-PCR performed on total RNA from patient's blood with a specific primer pair encompassing exons 6-8, which demonstrated that the variant creates a new splice acceptor site leading to the retention of the last 6 bases of intron 7 (r.736_737insTTGTAG) and consequent inframe insertion of 2 valine residues (p.Asn245_Gly246insValVal).

development was normal. At 4 years, the patient presented height $91 \mathrm{~cm}(-2.3 \mathrm{SD})$, weight $14 \mathrm{~kg}(-1.0 \mathrm{SD})$, OFC 49 cm (-0.39 SD), facial dysmorphism (Fig. 1a, b), short fingers, bilateral flatfoot, generalized joint hypermobility (Beighton score 9/9) (Fig. 1c), mild pectus excavatum, protruding abdomen with accentuated lumbar hyperlordosis, extremely soft and velvety skin, dystrophic postsurgical thoracic scars, and mild hypotonia. X-rays of hands (Fig. 1d) and feet excluded any carpal-tarsal synostoses. Spine magnetic resonance imaging showed multiple cervical intervertebral disk hernias and posterior vertebral segmentation defects (Fig. 1e). She never complained of otitis media, but a recent audiometric evaluation showed transmission deafness of mild degree with absence of the stapedial reflex. Ophthalmological survey gave normal results. Growth parameters and facial gestalt (Fig. 1f) remained unchanged at 6 and 6/12 years. Previous molecular investigations, all with negative/normal results, included CGH-array, single-nucleotide polymorphism b Schematic of MAP $3 K 7$ structure and protein domains (in blue the kinase domain (36-291) and in orange the TAB2-binding domain (BD) (506-574) according to ref. [4]). bMAP3K7 variants found in FMD2 (in blue, from ref. [9]) and CSCFS (in black, from ref. [8]) are below the diagram. The variant affecting function identified in this study is in red. Variants are annotated according to HGVS nomenclature (http://www. hgvs.org/mutnomen; NG_011966.2, NM_145331.2, NP_663304.1) (color figure online)

array, and targeted next-generation sequencing panels for coesinopathy and RASopathy genes. The phenotypic overlap with an our previous patient with a $T A B 2$ intragenic variant presumably affecting function (Fig. 1g) [14] and others described with the $T A B 2$ microdeletion syndrome [15] prompted us to investigate for variants in $T A B 2$ and MAP3K7.

\section{Molecular findings}

Molecular analysis did not reveal any variant in $T A B 2$ but disclosed the novel de novo c.737-7A $>\mathrm{G}$ variant in MAP3K7 (NM_145331.2; NG_011966.2: g.91261905T>C). The variant was predicted, in silico, to affect function by the creation of a new splice acceptor site with the retention of the last 6 bases of intron 7 and an in-frame insertion of 2 amino acid residues as a consequence (p.Asn245_Gly246insValVal) (NP_663304.1). RT-PCR on cDNA from patient's whole blood confirmed this prediction with the 
insertion of six bases at the RNA level (r.736_737insTTGTAG; Fig. 2a). The identified variant was not found in 500 in-house exome data from ethnicitymatched samples and from the Exome Aggregation Consortium (ExAC) browser.

\section{Discussion}

Molecular findings in this patient are in line with the diagnosis of CSFCS. The syndrome was first delineated in 2010 and then (2016) associated with MAP3K7 heterozygous variants affecting function in four pedigrees [8, 10]. In particular, the missense variants c.328G $>\mathrm{T}$ (p.(Gly110Cys)) and c.721T $>\mathrm{A}$ (p.(Trp241Arg)) and the in-frame deletion c.130_135del (p. (Arg44_Gly45del)) were found in three sporadic patients and the c.148_150del (p.(Val50del)) variant in a familial case [8]. All these variants fall within the kinase domain of TAK1 and patients share short stature, facial gestalt, mixed hearing loss, upper gut transitory dysfunction, congenital heart valve dysplasia, joint hypermobility, and cervical and carpal-tarsal fusions [8]. Considering the absence of any phenotypic overlap with genomic deletions encompassing $M A P 3 K 7$ [16], a stringent genotype-phenotype correlation underlying CSFCS is presumed. In line with previous findings, the variant r.736_737insTTGTAG (p.Asn245_Gly246insValVal) identified in our patient affects the TAK1 kinase domain (Fig. 2b). The localization of the identified variant, its rarity, the effect at the mRNA level, and the specificity of the associated phenotype supports its role as a disease-causing nucleotide change.

Table 1 compares clinical findings of all CSFCS patients (comprising the present case), our patient with TAB2 heterozygous frameshift variant, and a summary of patients with FMD type 2 and 3. Our patient with CSFCS lacks carpal-tarsal fusions and presents vertebral fusions extending to the posterior arches, a feature causing an unusual posture with extreme lumbar hyperlordosis. In this individual, the combination of soft connective tissue abnormalities prompted the pediatrician to ask for an evaluation in a specialized clinics. Similarities with a previously reported individual with $T A B 2$ heterozygous frameshift variant (Fig. 1f, g), and the negative results of other genetic testing suggested to sequence $T A B 2$ and, subsequently, $M A P 3 K 7$; the latter supported with the severe upper gastrointestinal dysmotility. While TAB2 point mutations seem rare, a microdeletion syndrome encompassing this gene has been recently described in multiple patients [15]. The phenotype associated with the TAB2microdeletion syndrome is wide and the involvement of neighboring genes is likely the cause of intellectual disability and other satellite symptoms. Our two patients with $M A P 3 K 7$ and $T A B 2$ intragenic variants share the overall

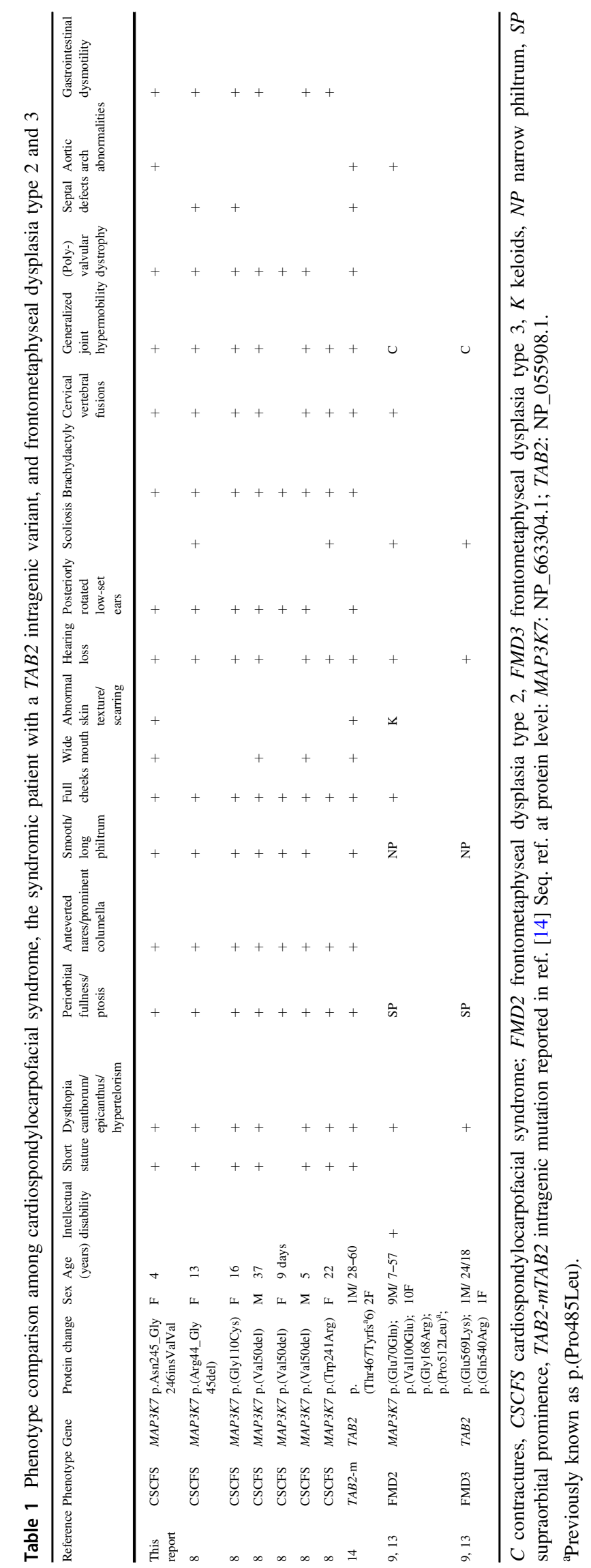


facial dysmorphology, short stature, and connective tissue abnormalities, and this might mirror the interactions of the two encoded proteins. A similar combination of features has been also recently described in a pedigree with FLNA intragenic variant with marked intrafamilial variability and lethal valvular dystrophy [17]. Further observations and function studies are needed in order to confirm and refine such a preliminary impression.

In summary, we report an additional patient with CSFCS due to a novel heterozygous in-frame duplication in MAP3K7. Our observation expands the CSFCS phenotype and the mutational spectrum of MAP3K7. The clinical similarities that we observed with other single families with point variants in TAB2 [14] and FLNA [17] could expand the complex molecular interactions among these genes, as recently demonstrated by the identification of the FMD allelic series. This study reinforces the role of TAK1-dependent signaling pathway in human morphogenesis and the existence of a community of syndromes linked to variants in different mediators of the related signaling transduction.

\section{Compliance with ethical standards}

Conflict of interest The authors declare that they have no conflict of interest.

\section{References}

1. Landström M. The TAK1-TRAF6 signalling pathway. Int J Biochem Cell Biol. 2010;42:585-9.

2. Koitabashi N, Danner T, Zaiman AL, et al. Pivotal role of cardiomyocyte TGF- $\beta$ signaling in the murine pathological response to sustained pressure overload. J Clin Invest. 2011;121:2301-12.

3. Morioka S, Inagaki M, Komatsu Y, Mishina Y, Matsumoto K, Ninomiya-Tsuji J. TAK1 kinase signaling regulates embryonic angiogenesis by modulating endothelial cell survival and migration. Blood. 2012;120:3846-57.

4. Besse A, Lamothe B, Campos AD, et al. TAK1-dependent signaling requires functional interaction with TAB2/TAB3. J Biol Chem. 2007;282:3918-28.

5. Morrison DK. MAP kinase pathways. Cold Spring Harb Perspect Biol. 2012;4:a011254. pii
6. Takaesu G, Kishida S, Hiyama A, et al. TAB2, a novel adaptor protein, mediates activation of TAK1 MAPKKK by linking TAK1 to TRAF6 in the IL-1 signal transduction pathway. Mol Cell. 2000;5:649-58.

7. Broglie P, Matsumoto K, Akira S, Brautigan DL, Ninomiya-Tsuji J. Transforming growth factor beta-activated kinase 1 (TAK1) kinase adaptor, TAK1-binding protein 2, plays dual roles in TAK1 signaling by recruiting both an activator and an inhibitor of TAK1 kinase in tumor necrosis factor signaling pathway. J Biol Chem. 2010;285:2333-9.

8. Le Goff C, Rogers C, Le Goff W, et al. Heterozygous mutations in MAP3K7, encoding TGF- $\beta$-activated kinase 1 , cause cardiospondylocarpofacial syndrome. Am J Hum Genet. 2016;99: 407-13.

9. Wade EM, Daniel PB, Jenkins ZA, et al. Mutations in MAP3K7 that alter the activity of the TAK1 signaling complex cause frontometaphyseal dysplasia. Am J Hum Genet. 2016;99: 392-406.

10. Sousa SB, Baujat G, Abadie V, et al. Postnatal growth retardation, facial dysmorphism, spondylocarpal synostosis, cardiac defect, and inner ear malformation (cardiospondylocarpofacial syndrome?)-a distinct syndrome? Am J Med Genet A. 2010;152A:539-46.

11. Robertson SP, Twigg SR, Sutherland-Smith AJ, et al. Localized mutations in the gene encoding the cytoskeletal protein filamin A cause diverse malformations in humans. Nat Genet. 2003;33: 487-91.

12. Martínez F, Caro-Llopis A, Roselló M, et al. High diagnostic yield of syndromic intellectual disability by targeted next-generation sequencing. J Med Genet. 2017;54:87-92.

13. Wade EM, Jenkins ZA, Daniel PB, et al. Autosomal dominant frontometaphyseal dysplasia: delineation of the clinical phenotype. Am J Med Genet A. 2017 (in press) https://doi.org/10. 1002/ajmg.a.38267.

14. Ritelli M, Morlino S, Giacopuzzi E, et al. A recognizable systemic connective tissue disorder with polyvalvular heart dystrophy and dysmorphism associated with TAB2 mutations. Clin Genet. 2017 (in press) Clin Genet 2018; 93: 126-133.

15. Cheng A, Dinulos MBP, Neufeld-Kaiser W, et al. 6q25.1 (TAB2) microdeletion syndrome: congenital heart defects and cardiomyopathy. Am J Med Genet A. 2017 (in press) https://doi.org/10. 1002/ajmg.a.38254.

16. Klein OD, Cotter PD, Moore MW, et al. Interstitial deletions of chromosome 6q: genotype-phenotype correlation utilizing array CGH. Clin Genet. 2007;71:260-6.

17. Ritelli M, Morlino S, Giacopuzzi E, et al. Ehlers-Danlos syndrome with lethal cardiac valvular dystrophy in males carrying a novel splice mutation in FLNA. Am J Med Genet A. 2017;173: 169-76. 